\title{
Design of Gain Scheduling Control Using State Derivative Feedback
}

\author{
Lázaro Ismael Hardy Llins, ${ }^{1}$ Edvaldo Assunção, ${ }^{1}$ Marcelo C. M. Teixeira, ${ }^{1}$ Rodrigo Cardim, ${ }^{1}$ \\ Mario R. R. Cadalso, ${ }^{1}$ Diogo R. de Oliveira, ${ }^{2}$ and Emerson R. P. da Silva ${ }^{3}$ \\ ${ }^{1}$ Department of Electrical Engineering, Ilha Solteira School of Engineering, São Paulo State University (UNESP), \\ Control Research Laboratory, José Carlos Rossi Ave. 1370, 15385-000 Ilha Solteira, SP, Brazil \\ ${ }^{2}$ Federal Institute of Education, Science and Technology of Mato Grosso do Sul (IFMS), Campus of Três Lagoas, \\ 79.641-162 Três Lagoas, MS, Brazil \\ ${ }^{3}$ Academic Department of Electrical Engineering, Federal Technological University of Paraná (UTFPR), \\ Alberto Carazzai Ave. 1640, 86300-000 Cornélio Procópio, PR, Brazil
}

Correspondence should be addressed to Lázaro Ismael Hardy Llins; hardyllins@gmail.com

Received 2 July 2017; Revised 10 November 2017; Accepted 19 November 2017; Published 17 December 2017

Academic Editor: Haranath Kar

Copyright (c) 2017 Lázaro Ismael Hardy Llins et al. This is an open access article distributed under the Creative Commons Attribution License, which permits unrestricted use, distribution, and reproduction in any medium, provided the original work is properly cited.

\begin{abstract}
In recent years, the study of systems subject to time-varying parameters has awakened the interest of many researchers. The gain scheduling control strategy guarantees a good performance for systems of this type and also is considered as the simplest to deal with problems of this nature. Moreover, the class of systems in which the state derivative signals are easier to obtain than the state signals, such as in the control for reducing vibrations in a mechanical system, has gained an important hole in control theory. Considering those ideas, we propose sufficient conditions via LMI for designing a gain scheduling controller using state derivative feedback. The D-stability methodology was used for improving the performance of the transitory response. Practical implementation in an active suspension system and comparison with other methods validates the efficiency of the proposed strategy.
\end{abstract}

\section{Introduction}

Since the emergence of dynamic systems control theory, the techniques most used to feedback systems are the output feedback or state feedback, but the use of accelerometers allowed opening a path for the study of derivative feedback of the state vector, due to the easy reconstruction of the derived signals to the signals themselves. For example, from the acceleration, velocity determination is a simple security integral, which presents no greater problem of experimental order [1].

The use of feedback from the state vector derivative (derivative feedback) for linear systems has been explored in recent years. Some researchers have sought to develop methods similar to those existing for the state vector feedback; for example, [1] developed a formula similar to the widespread Ackerman for linear systems (SISO) through derivative feedback. In [2], a new formulation was presented for the stabilization of linear multivariable systems under derivative feedback states. In [3], an analysis was presented of linear systems of observability and stability through the state vector derived and a study on the disturbance rejection with state derivative feedback. In [4], a geometric theory for dynamical systems with state derivative feedback for singular systems was shown. In [5], the theory was presented to the design of a less conservative controller for linear systems with polytopic uncertainties via derivative feedback ensuring the stability and robustness of the system. An approach to the stability and robustness with derivative feedback, including the weakness, can be seen in [6]. Still, in the literature, it is possible to find papers that include the use of derivative feedback in linear systems subject to uncertainties in the plant among others, using techniques based on linear matrix inequalities (LMIs).

The gain scheduling controller has motivated several studies in the field of control engineering. This control 
strategy is very popular for linear application in multiple fields such as aeronautics, military, and civil. The origin of the gain scheduling controllers took place in the 60s with the classic theory called gain scheduling based on linearization of a system on their equilibrium points [7-9].

The classical gain scheduling efficiency depends on the dynamic characteristics of the nonlinear system. These can be described as a combination of linear systems, which was associated with a linear controller for each operation point [10]. The controller is designed taking into account only the dynamics of the plant locally around an equilibrium point [10]. Classical gain scheduling controllers were applied intensely but had limitations. Working only in the area of the neighborhood of operating equilibrium points represented a technical deficiency. The gain scheduling controller does reduce the error but there is still a periodic part in the error signal of the system [11].

It can design the controller using methods based on standard $\mathscr{L}_{2}$ ensuring robustness and nominal stability of the system and improving the project gain scheduling [8]. Robust gain scheduler PI controllers are presented in [12]. It presented the procedure for obtaining the solutions via BMI. In [13] a methodology was described to generalize robust gain-scheduled PID controller design for affine LPV systems with polytopic uncertainty via BMI.

Fuzzy gain scheduling overcomes the disadvantages of classical gain scheduling, considering the stability restriction and performance in both the local and the global behavior. Technical fuzzy gain scheduling may involve classical scheduling gain as well as the LPV techniques [14]. Reference [15] presents a fuzzy gain scheduling of proportional integral derivative (FGS-PID) controller for guaranteeing stability of a multimachine power system. The bee colony optimization method was used to determine control rules of the FGS-PID. Reference [16] developed a methodology to the nonfragile $\mathscr{H}_{\infty}$ control problem for a class of discrete-time TakagiSugeno fuzzy systems with both randomly occurring gain variations and channel fading via LMI.

An important consideration in the design of a linear controller for a closed-loop system with uncertainty is the robustness and performance. When uncertainties are invariant or slow variations, the problem can be solved by using robust control techniques [17]. Sometimes, one can have considerable variations in the parameters; in this case, a robust controller does not guarantee a good performance and the stabilization of the plant by a controller designed assumes that the same is time invariant system. Whereas the variations of parameters can be measured during system operation, the gain scheduling strategy can provide more efficient solutions. The gain scheduling controllers are a function of a variant parameter of the plant. The controllers can be adjusted according to the variations in the dynamics of the plant. Therefore, in many applications such drivers' gain scheduling is more feasible than the robust controllers. The combination of both techniques has been studied in [18].

The main motivation of the work was to develop a control strategy using gain scheduling and state derivative feedback; both techniques are used for systems with time variant parameters. In recent years, the LMIs are used to solve a lot of control theory problems. It was interesting to propose a methodology for solving this class of problems of this type.

According to the authors' knowledge, there is no record of studies that consider the union of both control strategies. Our main challenge and objective were to develop and design a gain scheduling type control using derivative feedback that considers time-varying parameters to ensure stability and good system performance.

Notation. Throughout the text, the following notations are used: $\mathbb{K}_{r}=\{1,2, \ldots, r\}, r \in \mathbb{N} ; \operatorname{diag}\left\{M_{1}, M_{2}, \ldots, M_{r}\right\}$ represents a block-diagonal matrix in which the diagonal elements are $M_{1}, M_{2}, \ldots, M_{r} ; M>0$ is used to represent positive definite matrices and, equivalently, $M<0$ is used to represent negative definite matrices; $(\cdots)^{T}$ denotes a vector or matrix transposition; $(\cdots)^{-T}$ denotes the transposition of an inverted matrix; $(*)$ denotes the symmetric matrix.

Property 1 (see [19]). For all nonsymmetric $M$ matrix $(M \neq$ $\left.M^{T}\right)$, if $M+M^{T}<0$, then $M$ is invertible.

Property 2. One has

$$
\begin{aligned}
& F_{z}(\alpha(t))=\sum_{i=1}^{N} \alpha_{i}(t) F_{i}, \\
& F_{z z}(\alpha(t))=\sum_{i=1}^{N} \sum_{j=1}^{N} \alpha_{i}(t) \alpha_{j}(t) F_{i j}, \\
& \Lambda_{N}=\left\{\alpha(t) \in \mathbb{R}^{N}: \sum_{i=1}^{N} \alpha_{i}(t)=1, \alpha_{i}(t) \geq 0, i\right. \\
& \quad=1, \ldots, N\},
\end{aligned}
$$

such that $\alpha(t) \in \Lambda_{N}$ is a time-varying parameter. The condition $\sum_{i=1}^{N} \alpha_{i}(t)=1$ was used to obtain the convexity of the set and it was described by polytope vertices. The variable $\alpha_{i}(t)$ from $i=1, \ldots, N$ represents a convex combination of the vertices. In this case we can write the set using the minimum and maximum vertex values. This description in the unit simplex form provides the design of the gain scheduling controller using LMIs. For simplicity, $F_{z}(\alpha(t))$ will be denoted by $F_{z}$, similarly $F_{z z}(\alpha(t))$ by $F_{z z}$.

Property 3 (see [20]). If the following LMIs, $\Upsilon_{i i}<0$ for $i=$ $1,2, \ldots, N$ and $\Upsilon_{i j}+\Upsilon_{j i}<0$ for $1 \leq i<j \leq N$, are feasible, then the inequality $\sum_{i=1}^{N} \sum_{j=1}^{N} \alpha_{i} \alpha_{j} \Upsilon_{i j}=\Upsilon_{z z}<0$ holds, where $\alpha(t) \in \Lambda_{N}$.

\section{Design of Gain Scheduling Controller Using State Derivative Feedback}

In this section, a solution for linear systems with timevarying parameters is proposed, using the strategy of gain scheduling control using state derivative feedback. In this 
section, Finsler's Lemma is used to avoid cross product of uncertain matrices.

2.1. Finsler's Lemma, an LMI Formulation. Consider a linear system with time variant uncertainties, described by

$$
\begin{aligned}
\dot{x}(t) & =\sum_{i=1}^{N} \alpha_{i}(t) A_{i} x(t)+\sum_{i=1}^{N} \alpha_{i}(t) B_{i} u(t) \\
& =A_{z}(\alpha(t)) x(t)+B_{z}(\alpha(t)) u(t),
\end{aligned}
$$

where $A_{z}(\alpha(t)) \in \mathbb{R}^{n \times n}$ and $B_{z}(\alpha(t)) \in \mathbb{R}^{n \times m}$ are matrices that represent the time variant systems dynamics, $x(t) \in \mathbb{R}^{n}$ is the state vector, and $u(t) \in \mathbb{R}^{m}$ is the control input vector.

Using the compact notation of Property 2, (2) can be rewritten as

$$
\dot{x}(t)=A_{z} x(t)+B_{z} u(t) .
$$

The objective is to find a controller $K_{z}$, such that the feedback system with the control input is

$$
u(t)=-\sum_{j=1}^{N} \alpha_{j}(t) K_{j} \dot{x}(t)=-K_{z} \dot{x}(t) .
$$

In this case, the closed-loop system of (3) and (4) is obtained:

$$
\dot{x}(t)=A_{z} x(t)-B_{z} K_{z} \dot{x}(t) .
$$

or

$$
\dot{x}(t)=\left(I+B_{z} K_{z}\right)^{-1} A_{z} x(t) .
$$

If $A_{z}$ has null eigenvalue, then the feedback system, given by $\left(I+B_{z} K_{z}\right)^{-1} A_{z}$, has null eigenvalue. The controller $K_{z}$ can not modify the null eigenvalue of $A_{z}$, in this case $\operatorname{rank}[(I+$ $\left.\left.B_{z} K_{z}\right)^{-1} A_{z}\right]<n$.

For applying Finsler's Lemma, it was necessary to start from equality (7), like the result of the transformation (5):

$$
0=A_{z} x(t)-\left(I+B_{z} K_{z}\right) \dot{x}(t) .
$$

To obtain the LMIs for the design of the controllers, Lemma 1 is used. Using the notation of Property 2, consider that $\mathscr{B}_{z}=\mathscr{B}_{z}(\alpha(t))$.

Lemma 1 (Finsler's Lemma). Considering that $\mathscr{W} \in \mathbb{R}^{2 n}, \mathscr{D} \in$ $\mathbb{R}^{2 n \times 2 n}$ and $\mathscr{B}_{z} \in \mathbb{R}^{n \times 2 n}$ with $\operatorname{rank}\left(\mathscr{B}_{z}\right)<n$ and $\mathscr{B}_{z}^{\perp}$ is a base for the null space of $\mathscr{B}_{z}$ (i.e., $\mathscr{B}_{z} \mathscr{B}_{z}^{\perp}=0$ ).

So the next conditions are equivalent:

(i) $\mathscr{W}^{T} \mathscr{D} \mathscr{W}<0, \forall \mathscr{W} \neq 0, \mathscr{B}_{z} \mathscr{W}=0$,

(ii) $\mathscr{B}_{z}^{\perp^{T}} \mathscr{D} \mathscr{B}_{z}^{\perp}<0$,

(iii) $\exists \rho \in \mathbb{R}: \mathscr{D}-\rho \mathscr{B}_{z}^{T} \mathscr{B}_{z}<0$,

(iv) $\exists Q \in \mathbb{R}^{2 n \times n}: \mathscr{D}+\mathscr{Q} \mathscr{B}_{z}+\mathscr{B}_{z}^{T} Q^{T}<0$,

where $\rho$ and $Q$ are additional variables (or multipliers).
Proof. See [21].

Finsler's Lemma is widely used in many control applications or theory of stability analysis based on LMIs. This lemma ensures relaxation of the set of LMIs due to the disassociation of matrices or reduction of control design of the number of LMIs [22].

2.2. Controller Design with Stability Condition. Define the following vectors and matrices:

$$
\begin{aligned}
\mathscr{W} & =\left[\begin{array}{l}
x(t) \\
\dot{x}(t)
\end{array}\right], \\
\mathscr{D} & =\left[\begin{array}{ll}
0 & P \\
P & 0
\end{array}\right], \\
\mathscr{Q} & =\left[\begin{array}{l}
X \\
X
\end{array}\right], \\
\mathscr{B}_{z} & =\left[A_{z}-\left[I+B_{z} K_{z}\right]\right],
\end{aligned}
$$

where $X$ is a nonsingular matrix of appropriate dimensions.

From (8) and using items (i) and (iv) of Finsler's Lemma, in Theorem 2 sufficient conditions are proposed so that system (5) is stabilizable.

The following theorem was developed considering unique Lyapunov matrix $P$, and the objective was to simplify the methodology. In this case the derivative of parameter in function of time was not included, knowing that $\sum_{i=1}^{N} \dot{\alpha}_{i}(t)=$ 0 .

Theorem 2. Assuming $A_{z}$ is invertible, if there exist symmetric positive definite matrices $G \in \mathbb{R}^{n \times n}$, matrices $Z_{i}, Z_{j} \in \mathbb{R}^{m \times n}$ and $Q \in \mathbb{R}^{n \times n}$, with $i, j \in \mathbb{K}_{r}$, such that

$$
\begin{aligned}
& \Upsilon_{i i}<0, \quad i=1,2, \ldots, N, \\
& \Upsilon_{i j}+\Upsilon_{j i}<0, \quad 1 \leq i<j<N, \\
& \Upsilon_{i j}=\left[\begin{array}{cc}
A_{i} Q^{T}+Q A_{i}^{T} & G+Q A_{i}^{T}-Q^{T}-B_{j} Z_{i} \\
* & -Q^{T}-B_{i} Z_{j}-Q-Z_{j}^{T} B_{i}^{T}
\end{array}\right],
\end{aligned}
$$

where $\Upsilon_{i i}$ is a result of adopting $j=i$. Then system (5) is stabilizable and the gain matrices are given by

$$
K_{z}=Z_{z} Q^{-T}
$$

Proof. Consider that (9) and (10) are feasible. Then, applying Property 3 , observe that the following inequality holds:

$$
\left[\begin{array}{cc}
A_{z} Q^{T}+Q A_{z}^{T} & G+Q A_{z}^{T}-Q^{T}-B_{z} Z_{z} \\
* & -Q^{T}-B_{z} Z-Q-Z_{z}^{T} B_{z}^{T}
\end{array}\right]<0 .
$$

Replacing $Q=X^{-1}, G=X^{-1} P X^{-T}$, and $Z_{z}=K_{z} X^{-T}$, the following is obtained: 


$$
\left[\begin{array}{cc}
A_{z} X^{-T}+X^{-1} A_{z}^{T} & X^{-1} P X^{-T}+X^{-1} A_{z}^{T}-X^{-T}-B_{z} K_{z} X^{-T} \\
* & -X^{-T}-B_{z} K_{z} X^{-T}-X^{-1}-\left(K_{z} X^{-T}\right)^{T} B_{z}^{T}
\end{array}\right]<0
$$

Multiplying (14) to the left for $\left[\begin{array}{ll}X & X\end{array}\right]$ and to the right $\left[\begin{array}{ll}X & X\end{array}\right]^{T}$, one obtains

$$
\begin{aligned}
& {\left[\begin{array}{cc}
X A_{z}+A_{z}^{T} X^{T} & P+A_{z}^{T} X^{T}-X-X B_{z} K_{z} \\
* & -X-X B_{z} K_{z}-X^{T}-K_{z}^{T} B_{z}^{T} X^{T}
\end{array}\right]} \\
& <0 .
\end{aligned}
$$

Separating in terms turns

$$
\begin{aligned}
& {\left[\begin{array}{ll}
0 & P \\
P & 0
\end{array}\right]+\left[\begin{array}{cc}
X A_{z} & -X\left[I+B_{z} K_{z}\right] \\
X A_{z}-X\left[I+B_{z} K_{z}\right]
\end{array}\right]} \\
& +\left[\begin{array}{c}
A_{z}^{T} X^{T} \\
A_{z}^{T} X^{T} \\
-\left[I+K_{z}^{T} B_{z}^{T}\right] X^{T}-\left[I+K_{z}^{T} B_{z}^{T}\right] X^{T}
\end{array}\right]<0 .
\end{aligned}
$$

The multiplication of matrix can be separated in multiplications of matrices:

$$
\begin{gathered}
{\left[\begin{array}{ll}
0 & P \\
P & 0
\end{array}\right]+\left[\begin{array}{l}
X \\
X
\end{array}\right]\left[A_{z}-\left[I+B_{z} K_{z}\right]\right]} \\
+\left[\begin{array}{c}
A_{z}^{T} \\
-\left[I+B_{z} K_{z}\right]^{T}
\end{array}\right]\left[\begin{array}{ll}
X^{T} & \left.X^{T}\right]<0,
\end{array}\right.
\end{gathered}
$$

then, inequality (17) has the form of Finsler's Lemma:

$$
\begin{aligned}
\mathscr{W} & =\left[\begin{array}{l}
x(t) \\
\dot{x}(t)
\end{array}\right], \\
\mathscr{D} & =\left[\begin{array}{ll}
0 & P \\
P & 0
\end{array}\right], \\
\mathscr{Q} & =\left[\begin{array}{l}
X \\
X
\end{array}\right], \\
\mathscr{B}_{z} & =\left[A_{z}-\left[I+B_{z} K_{z}\right]\right] .
\end{aligned}
$$

It satisfies item (i) of Finsler's Lemma, then there is a matrix $P=P^{T}>0$, accomplishing the Lyapunov conditions for system (2), taking into account the gain matrices (12), then system (5) is asymptotically stable.

Figure 1 shows a set $\mathscr{D}$ for allocating the eigenvalues of the system. It is limited for relations between the constraints that meet the parameters Decay Rate $(\gamma)$, Ratio $(r)$, and Angle $(\theta)$.
2.3. Controller Design with Stability Condition and Decay Rate. Given a real constant $\gamma>0$, one can impose a decay rate constraint as shown in Figure 1, where $\lambda$ are the eigenvalues of closed-loop (5).

The decay rate constraint is imposed to the closed-loop system (5), if condition (19) is satisfied for the entire trajectory $x(t) \neq 0$ of the system, $t \geq 0$ [19].

$$
\dot{V}(x(t))<-2 \gamma V(x(t))
$$

then we have

$$
\dot{x}(t)^{T} P x(t)+x(t)^{T} P \dot{x}(t)<-2 \gamma x(t)^{T} P x(t) .
$$

From (5),

$$
\dot{x}(t)=\left(I+B_{z} K_{z}\right)^{-1} A_{z} x(t) .
$$

Replacing (21) in (20), the consideration of the decay rate is equivalent to solution of

$$
\begin{aligned}
A_{z}^{T}\left(I+B_{z} K_{z}\right)^{-T} P+P\left(I+B_{z} K_{z}\right)^{-1} A_{z}<-2 \gamma P & \\
P & >0 .
\end{aligned}
$$

Considering Lemma 1, sufficient conditions for system (5) to be stabilizable with restrictions on the decay rate $\gamma>0$, and defining the following vectors and matrices,

$$
\begin{aligned}
\mathscr{W} & =\left[\begin{array}{l}
x(t) \\
\dot{x}(t)
\end{array}\right], \\
\mathscr{D} & =\left[\begin{array}{cc}
2 \gamma P & P \\
P & 0
\end{array}\right], \\
\mathscr{Q} & =\left[\begin{array}{l}
X \\
X
\end{array}\right], \\
\mathscr{B}_{z} & =\left[A_{z}-\left[I+B_{z} K_{z}\right]\right],
\end{aligned}
$$

where $X$ is a nonsingular matrix of appropriate dimensions.

Using the definitions given in (23), in Theorem 3 sufficient conditions are proposed for system (5) to be stabilizable with decay rate $\gamma>0$.

Theorem 3. Assuming $A_{z}$ is invertible, if there exist symmetric positive definite matrices $G \in \mathbb{R}^{n \times n}$, matrices $Z_{i}, Z_{j} \in \mathbb{R}^{m \times n}$ and $Q \in \mathbb{R}^{n \times n}$, with $i, j \in \mathbb{K}_{r}$, such that

$$
\begin{aligned}
& \Upsilon_{i i}^{\gamma}<0, \quad i=1,2, \ldots, N, \\
& \Upsilon_{i j}^{\gamma}+\Upsilon_{j i}^{\gamma}<0, \quad 1 \leq i<j<N,
\end{aligned}
$$




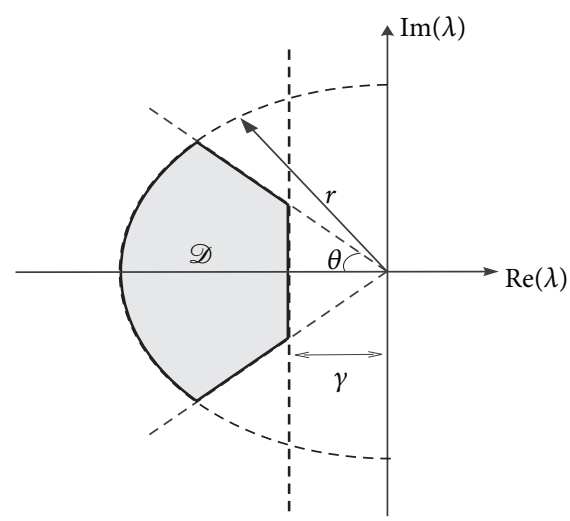

FIGURE 1: Region D-stability for pole placement [5].

$$
\begin{aligned}
& \Upsilon_{i j}^{\gamma} \\
& =\left[\begin{array}{cc}
2 \gamma G+A_{i} Q^{T}+Q A_{i}^{T} & G+Q A_{i}^{T}-Q^{T}-B_{i} Z_{j} \\
* & -Q^{T}-B_{i} Z_{j}-Q-Z_{j}^{T} B_{i}^{T}
\end{array}\right],
\end{aligned}
$$

where $Y_{i i}^{\gamma}$ is a result of adopting $j=i$. Then system (5) is stabilizable, with decay rate greater than or equal to $\gamma$, and the matrices of controller are given by

$$
K_{z}=Z_{z} Q^{-T} \text {. }
$$

Proof. The demonstration follows steps similar to the proof of Theorem 2, considering the condition of stability with restriction of decay rate (22).

2.4. Controller Design with Stability Condition, Decay Rate, and Less Conservative Solutions (Using $\kappa$ ). In the previous subsection the controller design using Finsler's Lemma considering the decay rate presents solutions that can be improved; given the empirical results, the value of $\gamma$ is limited. Applying a similar strategy, but incorporating a scalar value $\kappa>0$ into the inequalities, one can obtain less conservative solutions, obviously with an adequate value of $\kappa$ [5].

Considering Lemma 1 (Finsler's Lemma), sufficient conditions for system (2) to be stabilized with restrictions on the decay rates $\gamma>0, \kappa>0$, and defining the following vectors and matrices,

$$
\begin{aligned}
\mathscr{W} & =\left[\begin{array}{c}
x(t) \\
\dot{x}(t)
\end{array}\right], \\
\mathscr{D} & =\left[\begin{array}{cc}
2 \gamma P & P \\
P & 0
\end{array}\right], \\
\mathbb{Q} & =\left[\begin{array}{c}
X \\
\kappa X
\end{array}\right], \\
\mathscr{B}_{z} & =\left[A_{z}-\left[I+B_{z} K_{z}\right]\right],
\end{aligned}
$$

where $X$ is a nonsingular matrix of appropriate dimensions.
From the definitions given in (26), in Theorem 4 sufficient conditions are proposed for system (5) to be stabilizable with decay rates $\gamma>0$ and $\kappa>0$.

Theorem 4. Assuming $A_{z}$ is invertible, for given $\gamma$ and $\kappa$, if there exists a positive definite symmetric matrix $G \in \mathbb{R}^{n \times n}$, matrices $Z_{i}, Z_{j} \in \mathbb{R}^{m \times n}$ and $Q \in \mathbb{R}^{n \times n}$ such that

$$
\begin{aligned}
& Y_{i i}^{\gamma \kappa}<0, \quad i=1,2, \ldots, N, \\
& \Upsilon_{i j}^{\gamma \kappa}+\Upsilon_{j i}^{\gamma}<0, \quad 1 \leq i<j<N, \\
& \Upsilon_{i j}^{\gamma \kappa} \\
& =\left[\begin{array}{cc}
2 \gamma G+A_{i} Q^{T}+Q A_{i}^{T} & G+\kappa Q A_{i}^{T}-Q^{T}-B_{j} Z_{i} \\
* & -\kappa\left(Q^{T}+B_{i} Z_{j}+Q+Z_{j}^{T} B_{i}^{T}\right)
\end{array}\right],
\end{aligned}
$$

where $Y_{i i}^{\gamma \kappa}$ is a result of adopting $j=i$. Then system (5) is stabilizable, with decay rate greater than or equal to $\gamma$, and the matrices of controller are given by

$$
K_{z}=Z_{z} Q^{-T} .
$$

Proof. It is similar to the proof of Theorem 2.

\subsection{Controller Design with Stability Condition and Radius Restriction $r$}

Theorem 5. Assuming $A_{z}$ is invertible, for a given $r$, if there exists a symmetric positive defined matrix $G \in \mathbb{R}^{n \times n}$, matrices $Z_{i}, Z_{j} \in \mathbb{R}^{m \times n}$ and $W \in \mathbb{R}^{n \times n}$ such that

$$
\begin{aligned}
& \Upsilon_{i i}^{r}<0, \quad i=1,2, \ldots, N, \\
& \Upsilon_{i j}^{r}+Y_{j i}^{r}<0, \quad 1 \leq i<j<N, \\
& Y_{i j}^{r}=\left[\begin{array}{crc}
\left(\begin{array}{c}
-r G+A_{i} W^{T} \\
+W A_{i}^{T}
\end{array}\right) & \left(\begin{array}{c}
-W^{T}-B_{i} Z_{j} \\
+W A_{i}^{T}
\end{array}\right) & 0_{n \times n} \\
* & \left(\begin{array}{c}
-W^{T}-B_{i} Z_{j} \\
-W-Z_{j}^{T} B_{i}^{T}
\end{array}\right) & G \\
* & -r G
\end{array}\right],
\end{aligned}
$$

where $Y_{i i}^{r}$ is a result of adopting $j=i$. Then system (5) is stabilizable with eigenvalues inside the circle of radius $r$, for all $\alpha(t)$. The gain matrices of controller are given:

$$
K_{z}=Z_{z} W^{-T} \text {. }
$$

Proof. From Property 3, if the LMIs (29)-(30) are feasible, then the inequality $\Upsilon_{z z}^{r}<0$ holds. Replacing $G=X^{-1} P X^{-T}$, $W=X^{-1}$, and $Z_{z}=K_{z} X^{-T}$ and multiplying $Y_{z z}^{r}$ to the left for $\operatorname{diag}(X, X, X, X)$ and to the right for $\operatorname{diag}\left(X^{T}, X^{T}, X^{T}, X^{T}\right)$, the following is obtained:

$$
\left[\begin{array}{ccc}
\left(\begin{array}{c}
-r P+X A_{z} \\
+A_{z}^{T} X^{T}
\end{array}\right) & \left(\begin{array}{c}
-X+A_{z}^{T} X^{T} \\
-X B_{z} K_{z}
\end{array}\right) & 0_{n \times n} \\
* & \left(\begin{array}{c}
-X-X B_{z} K_{z} \\
-X^{T}-K_{z}^{T} B_{z}^{T} X^{T}
\end{array}\right) & P \\
* & * & -r P
\end{array}\right]<0 .
$$


From $-X-X^{T}-X B_{z} K_{z}-K_{z}^{T} B_{z}^{T} X^{T}<0 \Leftrightarrow X\left(I+B_{z} K_{z}\right)+$ $\left(I+B_{z} K_{z}\right)^{T} X^{T}>0$ and according to Property 1 , conclude that $X\left(I+B_{z} K_{z}\right)$ is invertible, then $\left(I+B_{z} K_{z}\right)$ and $X$ are invertible. From item (iv) of Lemma 1, inequality (33) can be rewritten as $\mathscr{D}+\mathscr{Q} \mathscr{B}_{z}+\mathscr{B}_{z}^{T} \mathscr{Q}^{T}<0$, where

$$
\begin{aligned}
\mathscr{D} & =\left[\begin{array}{ccc}
-r P & 0_{n \times n} & 0_{n \times n} \\
* & 0_{n \times n} & P \\
* & * & -r P
\end{array}\right], \\
\mathscr{Q} & =\left[\begin{array}{c}
X \\
X \\
0_{n \times n}
\end{array}\right] \\
\mathscr{B}_{z} & =\left[\begin{array}{lll}
A_{z}-\left(I+B_{z} K_{z}\right) & 0_{n \times n}
\end{array}\right] .
\end{aligned}
$$

Now consider that

$$
\mathscr{B}_{z}^{\perp}=\left[\begin{array}{cc}
A_{z}^{-1} & 0_{n \times n} \\
\left(I+B_{z} K_{z}\right)^{-1} & 0_{n \times n} \\
0_{n \times n} & I
\end{array}\right] .
$$

Note that $\mathscr{B}_{z} \mathscr{B}_{z}^{\perp}=0_{n \times 2 n}$. Then, from (34)-(35) and considering item (ii) of Lemma 1, the equivalence is established:

$$
\begin{aligned}
\mathscr{B}_{z}^{\perp^{T}} \mathscr{D} \mathscr{B}_{z}^{\perp} & =\left[\begin{array}{cc}
-A_{z}^{-T} r P A_{z}^{-1} & \left(I+B_{z} K_{z}\right)^{-T} P \\
P\left(I+B_{z} K_{z}\right)^{-1} & -r P
\end{array}\right] \\
& <0,
\end{aligned}
$$

multiplying (36) to the left for $\operatorname{diag}\left(A_{z}^{T}, I\right)$ and to the right for $\operatorname{diag}\left(A_{z}, I\right)$, the following is obtained:

$$
\left[\begin{array}{cc}
r P & A_{N}^{T} P \\
P A_{N} & r P
\end{array}\right]<0
$$

where

$$
A_{N}=\left(I+B_{z} K_{z}\right)^{-1} A_{z},
$$

and the proof is completed.

In the next section, the LMIs conditions are proposed for inclusion of the restriction of the angle of $\mathscr{D}$-stability.

\subsection{Controller Design with Stability Condition and Angle Restriction $\theta$}

Theorem 6. Assuming $A_{z}$ is invertible, for a given $\theta$, if there exists a symmetric positive defined matrix $G \in \mathbb{R}^{n \times n}$, matrices $Z_{i}, Z_{j} \in \mathbb{R}^{m \times n}$ and $W \in \mathbb{R}^{n \times n}$ such that

$$
\Upsilon_{i i}^{\theta}<0, \quad i=1,2, \ldots, N
$$

$$
\begin{aligned}
& \Upsilon_{i j}^{\theta}+\Upsilon_{j i}^{\theta}<0, \quad 1 \leq i<j<N, \\
& \Upsilon_{i j}^{\theta}=\left[\begin{array}{llll}
M_{11}^{i j} & M_{12}^{i j} & M_{13}^{i j} & M_{14}^{i j}
\end{array}\right], \\
& M_{11}^{i j}=\left[\begin{array}{c}
\sin (\theta)\left(A_{i} W^{T}+W A_{i}^{T}\right) \\
\sin (\theta)\left(G-W+A_{i} W^{T}-Z_{j}^{T} B_{i}^{T}\right) \\
\cos (\theta)\left(G+A_{i} W^{T}+W+Z_{j}^{T} B_{i}^{T}\right) \\
\cos (\theta)\left(A_{i} W^{T}-W A_{i}^{T}\right)
\end{array}\right], \\
& M_{12}^{i j}=\left[\begin{array}{c}
\sin (\theta)\left(G-W^{T}+W A_{i}^{T}-B_{i} Z_{j}\right) \\
\sin (\theta)\left(-W^{T}-B_{i} Z_{j}-W-Z_{j}^{T} B_{i}^{T}\right) \\
\cos (\theta)\left(-W^{T}-B_{i} Z_{j}+W+Z_{j}^{T} B_{i}^{T}\right) \\
\cos (\theta)\left(-G-W^{T}-B_{i} Z_{j}-W A_{i}^{T}\right)
\end{array}\right], \\
& M_{13}^{i j}=\left[\begin{array}{c}
\cos (\theta)\left(G+W^{T}+W A_{i}^{T}+B_{i} Z_{j}\right) \\
\cos (\theta)\left(W^{T}+B_{i} Z_{j}-W-Z_{j}^{T} B_{i}^{T}\right) \\
\sin (\theta)\left(-W^{T}-B_{i} Z_{j}-W-Z_{j}^{T} B_{i}^{T}\right) \\
\sin (\theta)\left(G-W^{T}-B_{i} Z_{j}+W A_{i}^{T}\right)
\end{array}\right], \\
& M_{14}^{i j}=\left[\begin{array}{c}
\cos (\theta)\left(-A_{i} W^{T}+W A_{i}^{T}\right) \\
\cos (\theta)\left(-G-A_{i} W^{T}-W-Z_{j}^{T} B_{i}^{T}\right) \\
\sin (\theta)\left(G+A_{i} W^{T}-W-Z_{j}^{T} B_{i}^{T}\right) \\
\sin (\theta)\left(A_{i} W^{T}+W A_{i}^{T}\right)
\end{array}\right] \text {, }
\end{aligned}
$$

where $Y_{i i}^{r}$ is a result of adopting $j=i$. Then system (5) is stabilizable with eigenvalues, inside of angle $\theta$, for all $\alpha(t)$. The gain matrices of controller are given by

$$
K_{z}=Z_{z} W^{-T}
$$

Proof. From Property 3, if the LMIs (39)-(40) are feasible, then the inequality $\Upsilon_{z z}^{\theta}<0$ holds. Replacing $G=X^{-1} P X^{-T}$, $W=X^{-1}$, and $Z_{z}=K_{z} X^{-T}$ and multiplying $Y_{z z}^{\theta}$ to the left for $\operatorname{diag}(X, X, X, X)$ and to the right for $\operatorname{diag}\left(X^{T}, X^{T}, X^{T}, X^{T}\right)$, the following is obtained:

$$
\left[\begin{array}{llll}
M_{21} & M_{22} & M_{23} & M_{24}
\end{array}\right]<0,
$$

where

$$
M_{21}=\left[\begin{array}{c}
\sin (\theta)\left(X A_{z}+A_{z}^{T} X^{T}\right) \\
\sin (\theta)\left(P+X A_{z}-X^{T}-K_{z}^{T} B_{z}^{T} X^{T}\right) \\
\cos (\theta)\left(P+X A_{z}+X^{T}+K_{z}^{T} B_{z}^{T} X^{T}\right) \\
\cos (\theta)\left(X A_{z}-A_{z}^{T} X^{T}\right)
\end{array}\right] \text {, }
$$




$$
\begin{gathered}
M_{22}=\left[\begin{array}{c}
\sin (\theta)\left(P-X+A_{z}^{T} X^{T}-X B_{z} K_{z}\right) \\
\sin (\theta)\left(-X-X B_{z} K_{z}-X^{T}-K_{z}^{T} B_{z}^{T} X^{T}\right) \\
\cos (\theta)\left(-X-X B_{z} K_{z}+X^{T}+K_{z}^{T} B_{z}^{T} X^{T}\right) \\
\cos (\theta)\left(-P-X-X B_{z} K_{z}-A_{z}^{T} X^{T}\right)
\end{array}\right], \\
M_{23}=\left[\begin{array}{c}
\cos (\theta)\left(P+X+A_{z}^{T} X^{T}+X B_{z} K_{z}\right) \\
\cos (\theta)\left(X+X B_{z} K_{z}-X^{T}-K_{z}^{T} B_{z}^{T} X^{T}\right) \\
\sin (\theta)\left(-X-X B_{z} K_{z}-X^{T}-K_{z}^{T} B_{z}^{T} X^{T}\right) \\
\sin (\theta)\left(P-X-X B_{z} K_{z}+A_{z}^{T} X^{T}\right)
\end{array}\right], \\
M_{24}=\left[\begin{array}{c}
\cos (\theta)\left(-X A_{z}+A_{z}^{T} X^{T}\right) \\
\cos (\theta)\left(-P-X A_{z}-X^{T}-K_{z}^{T} B_{z}^{T} X^{T}\right) \\
\sin (\theta)\left(P+X A_{z}-X^{T}-K_{z}^{T} B_{z}^{T} X^{T}\right) \\
\sin (\theta)\left(X A_{z}+A_{z}^{T} X^{T}\right)
\end{array}\right] .
\end{gathered}
$$

From $-X-\mathrm{X}^{T}-X B_{z} K_{z}-K_{z}^{T} B_{z}^{T} X^{T}<0 \Leftrightarrow X\left(I+B_{z} K_{z}\right)+$ $\left(I+B_{z} K_{z}\right)^{T} X^{T}>0$ and according to Property 1 , conclude that $X\left(I+B_{z} K_{z}\right)$ is invertible, then $\left(I+B_{z} K_{z}\right)$ and $X$ are invertible. From item (iv) of Lemma 1, inequality (43) can be rewritten as $\mathscr{D}+\mathscr{Q} \mathscr{B}_{z}+\mathscr{B}_{z}^{T} \mathscr{Q}^{T}<0$, where

$$
\begin{aligned}
\mathscr{D} & =\left[\begin{array}{cccc}
0_{n \times n} & \sin (\theta) P & \cos (\theta) P & 0_{n \times n} \\
* & 0_{n \times n} & 0_{n \times n} & -\cos (\theta) P \\
* & * & 0_{n \times n} & \sin (\theta) P \\
* & * & * & 0_{n \times n}
\end{array}\right], \\
\mathscr{Q} & =\left[\begin{array}{cc}
\sin (\theta) X & -\cos (\theta) X \\
\sin (\theta) X & -\cos (\theta) X \\
\cos (\theta) X & \sin (\theta) X \\
\cos (\theta) X & \sin (\theta) X
\end{array}\right], \\
\mathscr{B}_{z} & =\left[\begin{array}{cccc}
A_{z} & -\left(I+B_{z} K_{z}\right) & 0_{n \times n} & 0_{n \times n} \\
0_{n \times n} & 0_{n \times n} & -\left(I+B_{z} K_{z}\right) & A_{z}
\end{array}\right] .
\end{aligned}
$$

Now consider that

$$
\mathscr{B}_{z}^{\perp^{T}}=\left[\begin{array}{cccc}
A_{z}^{-1} & \left(I+B_{z} K_{z}\right)^{-1} & 0_{n \times n} & 0_{n \times n} \\
0_{n \times n} & 0_{n \times n} & \left(I+B_{z} K_{z}\right)^{-1} & A_{z}^{-1}
\end{array}\right] .
$$

Note that $\mathscr{B}_{z} \mathscr{B}_{z}^{\perp}=0_{2 n \times 2 n}$. Then, from (45)-(46) and considering item (ii) of Lemma 1 , the equivalence is established:

$$
\mathscr{B}_{z}^{\perp^{T}} \mathscr{D}_{z}^{\perp}=\left[\begin{array}{cc}
\left(\begin{array}{c}
\sin (\theta)\left(I+B_{z} K_{z}\right)^{-T} P A_{z}^{-1} \\
+\sin (\theta) A_{z}^{-T} P\left(I+B_{z} K_{z}\right)^{-1}
\end{array}\right) & \left(\begin{array}{c}
\cos (\theta) A_{z}^{-T} P\left(I+B_{z} K_{z}\right)^{-1} \\
-\cos (\theta)\left(I+B_{z} K_{z}\right)^{-T} P A_{z}^{-1}
\end{array}\right) \\
\left(\begin{array}{c}
\cos (\theta) A_{z}^{-T} P\left(I+B_{z} K_{z}\right)^{-1} \\
+\cos (\theta)\left(I+B_{z} K_{z}\right)^{-T} P A_{z}^{-1}
\end{array}\right) & \left(\begin{array}{c}
\sin (\theta) A_{z}^{-T} P\left(I+B_{z} K_{z}\right)^{-1} \\
+\sin (\theta)\left(I+B_{z} K_{z}\right)^{-T} P A_{z}^{-1}
\end{array}\right)
\end{array}\right]<0 .
$$

Multiplying (47) to the left for $\operatorname{diag}\left(A_{z}^{T}, A_{z}^{T}\right)$ and to the right for $\operatorname{diag}\left(A_{z}, A_{z}\right)$, the following is obtained:

$$
\begin{aligned}
& {\left[\begin{array}{ll}
\sin (\theta)\left(A_{N}^{T} P+P A_{N}\right) & \cos (\theta)\left(P A_{N}-A_{N}^{T} P\right) \\
\cos (\theta)\left(A_{N}^{T} P-P A_{N}\right) & \sin (\theta)\left(P A_{N}+A_{N}^{T} P\right)
\end{array}\right]} \\
& \quad<0 ;
\end{aligned}
$$

where

$$
A_{N}=\left(I+B_{z} K_{z}\right)^{-1} A_{z},
$$

then the proof is completed.

\section{Active Suspension System of a Vehicle}

The laboratory of active suspension, built by Quanser, can be represented in Figure 2. The schematic model of active suspension system is presented in Figure 3.

The system has a set consisting of two masses, called $M_{s}$ and $M_{u s}$. The mass $M_{s}$ represents $1 / 4$ of the total vehicle body and is supported by the spring $k_{s}$ and the damper $b_{s}$. The mass $M_{u s}$ corresponds to the mass of the vehicle and tire assembly is supported by the spring $k_{u s}$ and by the damper $b_{u s}$.
In order to diminish the vibrations caused by irregularities in the track the active suspension system is used, represented by a motor (actuator) connected between the masses $M_{s}$ and $M_{u s}$ and controlled by force $F_{c}$. In Table 1 the values of parameters are represented.

The schematic model [23] can be represented in state space, like what is shown in

$$
\begin{aligned}
\dot{x}(t)= & {\left[\begin{array}{cccc}
0 & 1 & 0 & -1 \\
\frac{-k_{s}}{M_{s}} & \frac{-b_{s}}{M_{s}} & 0 & \frac{b_{s}}{M_{s}} \\
0 & 0 & 0 & 1 \\
\frac{-k_{s}}{M_{u s}} & \frac{b_{s}}{M_{u s}} & \frac{-k_{u s}}{M_{u s}} & \frac{-\left(b_{s}+b_{u s}\right)}{M_{u s}}
\end{array}\right] x(t) } \\
& +\left[\begin{array}{c}
0 \\
\frac{1}{M_{s}} \\
0 \\
\frac{-1}{M_{u s}}
\end{array}\right] u(t),
\end{aligned}
$$


TABLE 1: Parameters of the active suspension.

\begin{tabular}{lc}
\hline Symbol & Value \\
\hline$M_{s}$ & $2.45(\mathrm{~kg})$ \\
$M_{u s}$ & $1(\mathrm{~kg})$ \\
$K_{s}$ & $900(\mathrm{~N} / \mathrm{m})$ \\
$k_{u s}$ & $2500(\mathrm{~N} / \mathrm{m})$ \\
$b_{s}$ & $7.5(\mathrm{Ns} / \mathrm{m})$ \\
$b_{u s}$ & $5(\mathrm{Ns} / \mathrm{m})$ \\
\hline
\end{tabular}

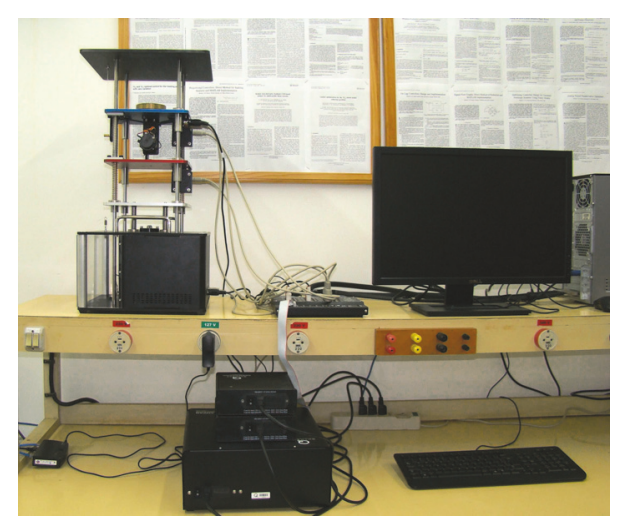

Figure 2: Active suspension system of the Quanser, belonging to LPC-FEIS-UNESP.

$$
\begin{aligned}
& x(t)=\left[\begin{array}{c}
z_{s}(t)-z_{u s}(t) \\
\dot{z}_{s}(t) \\
z_{u s}(t)-z_{r}(t) \\
\dot{z}_{u s}(t)
\end{array}\right], \\
& y(t)=\left[\begin{array}{llll}
1 & 0 & 0 & 0 \\
0 & 0 & 1 & 0
\end{array}\right] x(t),
\end{aligned}
$$

where $x_{2}(t)=\dot{z}_{s}(t)$ and $x_{4}(t)=\dot{z}_{u s}(t)$, representing the respective velocities of each mass.

For accomplishing the control design of active suspension, it was considered that the system has the amplified control signal, producing a variation on the input vector matrix. This variation is given taking into account the fault of the $40 \%$ of the amplified control signal. An actuator fault can be described as politopic uncertainties.

Therefore, to perform the control project, the following vertices of the polytope were considered:

$$
\begin{aligned}
& A_{1}=A_{2}=\left[\begin{array}{cccc}
0 & 1 & 0 & -1 \\
-367.347 & -3.061 & 0 & 3.061 \\
0 & 0 & 0 & 1 \\
900 & 7.5 & -2500 & -12.5
\end{array}\right], \\
& B_{1}=\left[\begin{array}{llll}
0 & -0.408 & 0 & -1
\end{array}\right]^{T}, \\
& B_{2}=\left[\begin{array}{llll}
0 & -0.244 & 0 & -0.6
\end{array}\right]^{T} .
\end{aligned}
$$

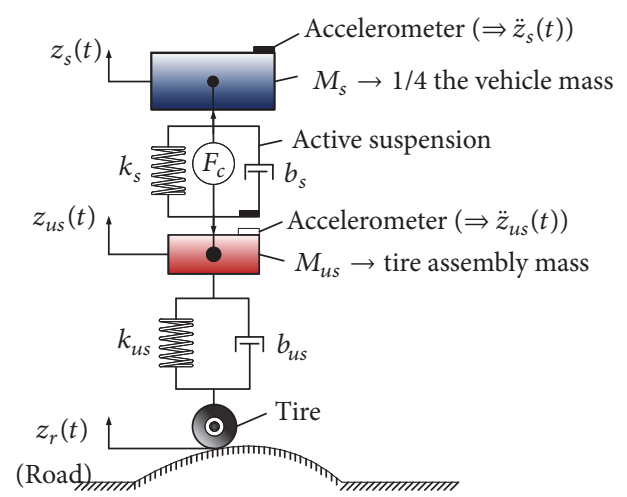

FIgURE 3: Schematic model of active suspension system.

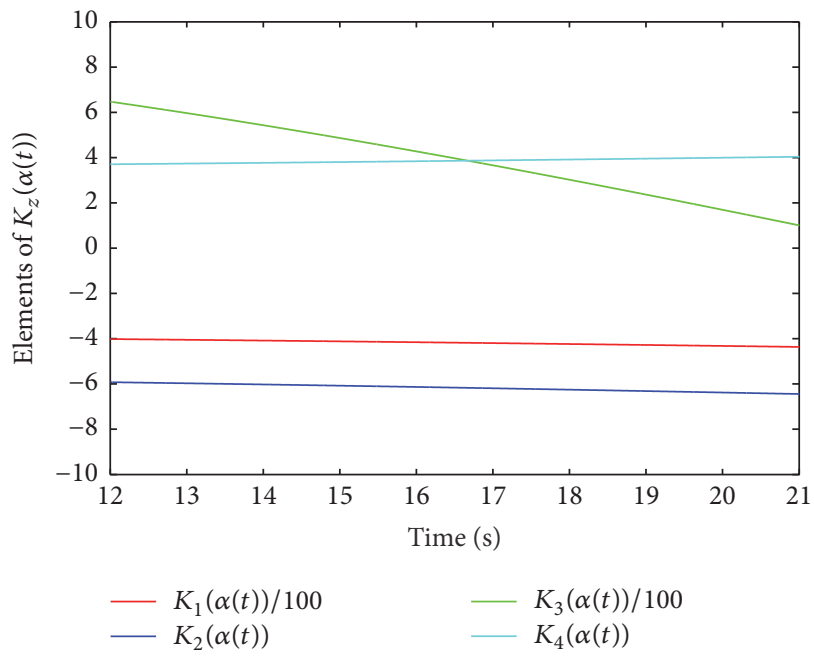

Figure 4: Elements of $K_{z}(\alpha(t))$ as function of time.

\section{Practical Implementation}

In this section, the practical implementation and results will be presented, with which it is possible to make a detailed analysis of the technique implemented.

For designing the values of the gain matrix, it was necessary to solve the LMI presented in Theorem 2:

$$
\begin{aligned}
& K_{d_{1}}=\left[\begin{array}{llll}
381.9988 & 5.6279 & -955.6624 & -3.5189
\end{array}\right], \\
& K_{d_{2}}=\left[\begin{array}{llll}
526.7000 & 7.8000 & -1320.0000 & -4.9000
\end{array}\right],
\end{aligned}
$$

and the value of $K_{z}(\alpha(t))$ shown in Figure 4 is given by the sum of the products of two sinusoidal functions $\alpha_{1}(t)$ shown in Figure 5 and $\alpha_{2}(t)$ which is the complement of the unit simplex.

The first practical implementation of the reference signal $z_{r}$ was chosen to reproduce a square wave $0.02 \mathrm{~m}$ amplitude, frequency $1 / 3 \mathrm{~Hz}$ pulse width $50 \%$. In the range of 0 to $12 \mathrm{~s}$ the system is open loop and in the range of 12.01 to $21 \mathrm{~s}$ the system is closed-loop. It is highlighted that in closed-loop used the control law is given by $u=K_{z}(\alpha(t)) \dot{x}$.

Figure 4 illustrates $K_{z}(\alpha(t))$ along time. 


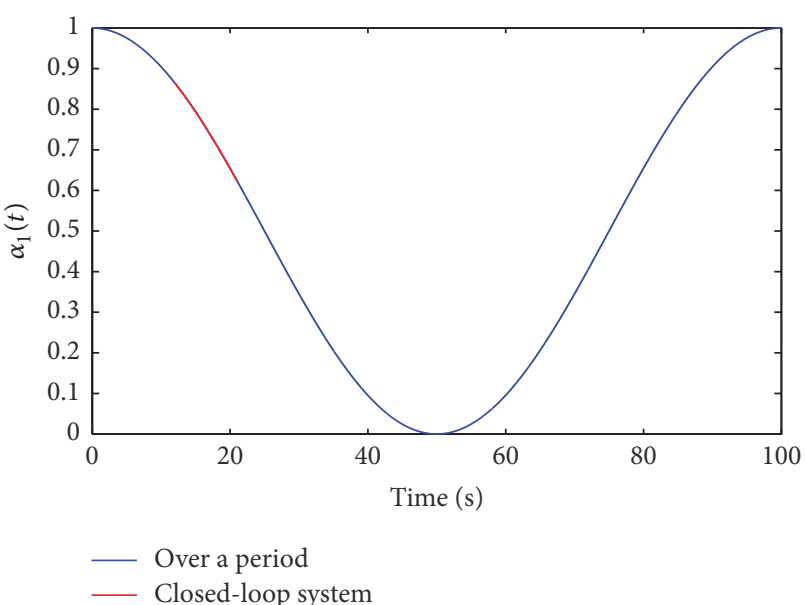

Figure 5: Sinusoidal function $\alpha_{1}(t)$.

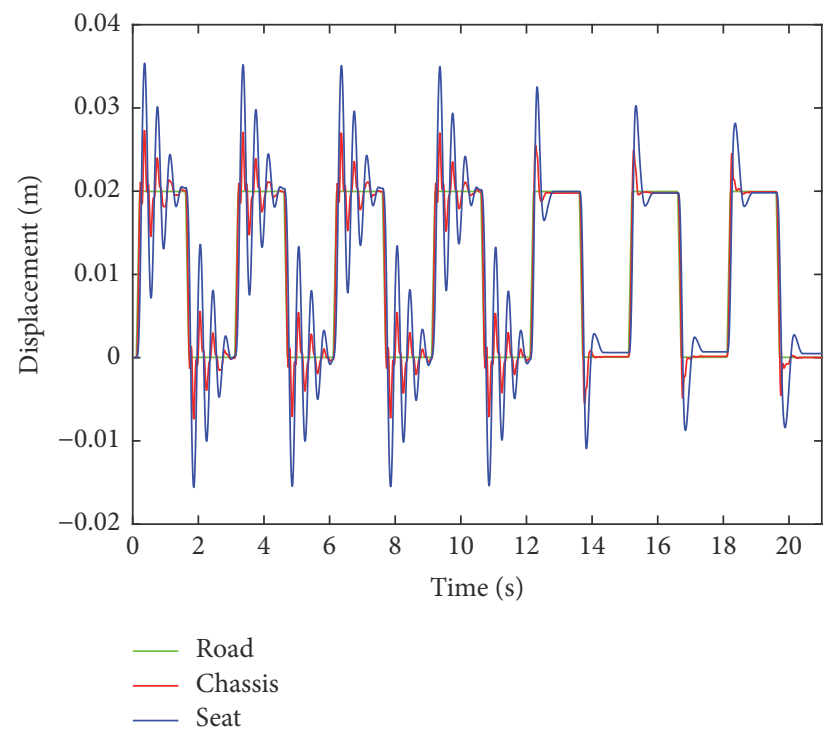

FIGURE 6: Transient response practice open loop (0-12 s) and closedloop (12.01-21 s).

In this case the controller is given by

$$
K_{z}(\alpha(t))=\alpha_{1}(t) K_{d_{1}}+\left(1-\alpha_{1}(t)\right) K_{d_{2}},
$$

where the variation of $\alpha_{1}(t)$ is illustrated in Figure 5, which highlights the period in which the system is closed-loop. As shown in Figure 6, the total time of the suspension drive was $21 \mathrm{~s}$, so it is possible to make the data acquisition to results analysis and display in a single graph.

Figure 10 shows how the open loop system tends to stability, although it has fluctuations that may affect the driver's comfort. As estimated, the closed-loop system ensures a good performance to the system, reducing the oscillations and the amplitude of the driver's seat displacement.

4.1. Derivate State Feedback Using D-Stability Constraints. The values of parameters $\gamma, r$, and $\theta$ were obtained considering the norm of controller gains. It was given for the allocation of eigenvalues.

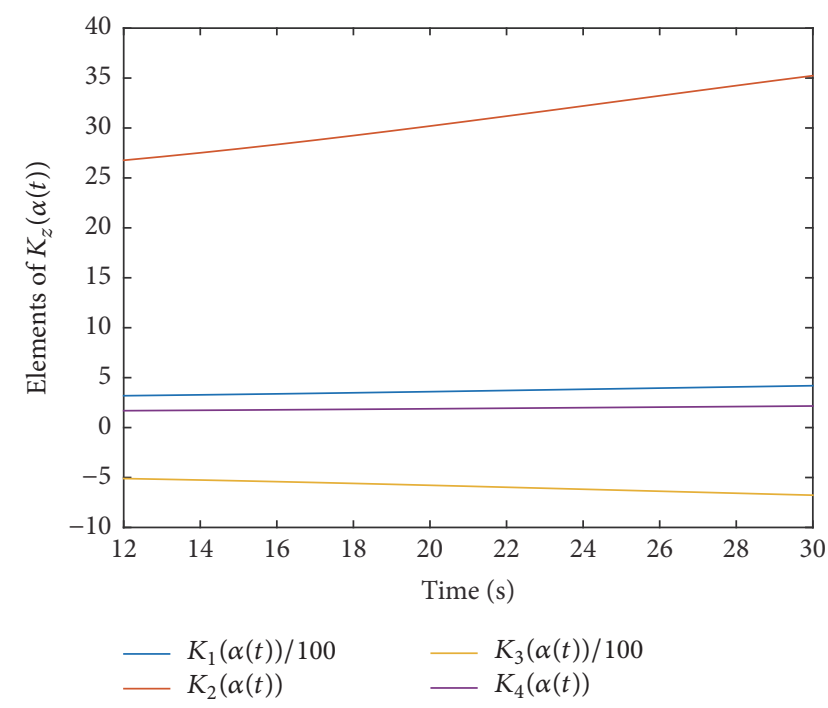

FIgURE 7: Elements of $K_{z}(\alpha(t))$ as a function of time.

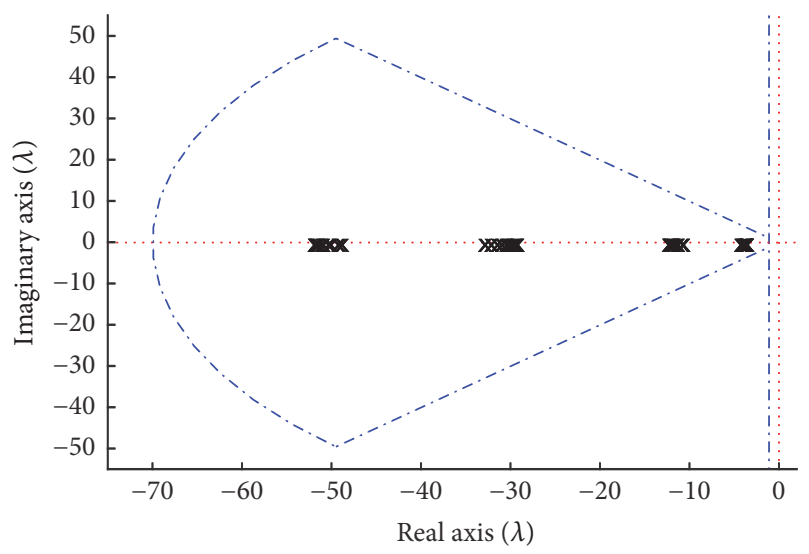

Figure 8: Placement of system eigenvalue cloud.

Using the set of LMIs of Theorems 4, 5, and 6 with $\gamma=$ $1.11, \kappa=0.9, r=70, \theta=45^{\circ}$, and parameter $\alpha(t)$ varying with frequency of $0.01 \mathrm{~Hz}$, the calculated gains were

$$
\begin{aligned}
& K_{d_{1}}=\left[\begin{array}{llll}
292.7180 & 24.5552 & -466.9545 & 1.5677
\end{array}\right], \\
& K_{d_{2}}=\left[\begin{array}{llll}
486.0575 & 40.8744 & -787.9186 & 2.4722
\end{array}\right] .
\end{aligned}
$$

The frequency of $\alpha(t)$ was adopted equal to $0.01 \mathrm{~Hz}$; in this case the time evolution of the elements of the controller is shown in Figure 7 to 30 seconds.

Figure 8 shows that the eigenvalues are inside $\mathscr{D}$-stability set for variations of $0 \leq \alpha(t) \leq 1$.

In Figure 9, it is noticed that the system presents a good dynamic performance which decreases overshoot of transient response, showing the efficiency of the new methodology.

In [24], there is no limitation of the gain of feedback which makes its practical implementation difficult; therefore this new proposal is more comprehensive.

4.2. Comparison Analysis. Figure 10 represents the transitory response considering the methodology proposed in [5]. 


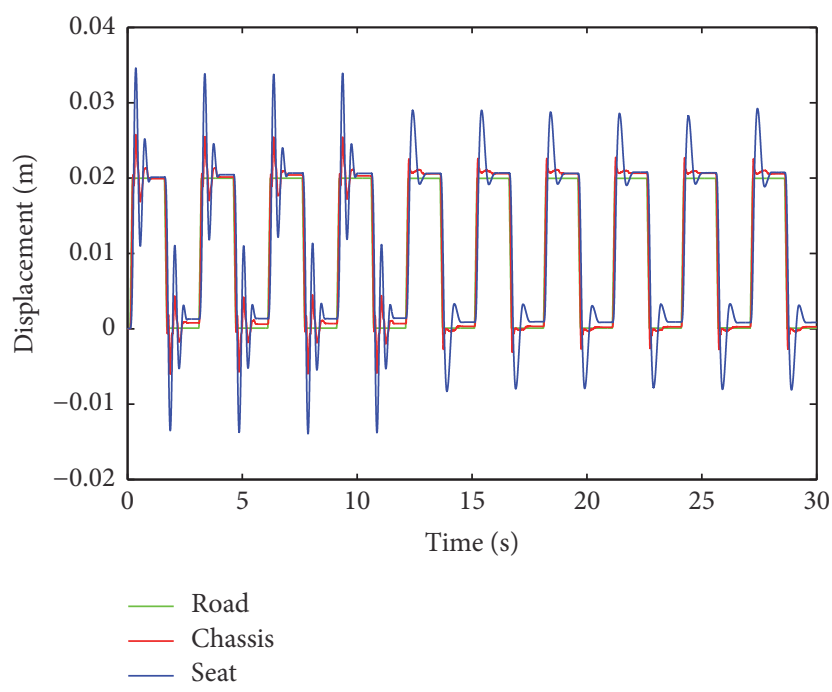

Figure 9: Transient response of open loop (0-12 s) and closed-loop (12.01-30 s).

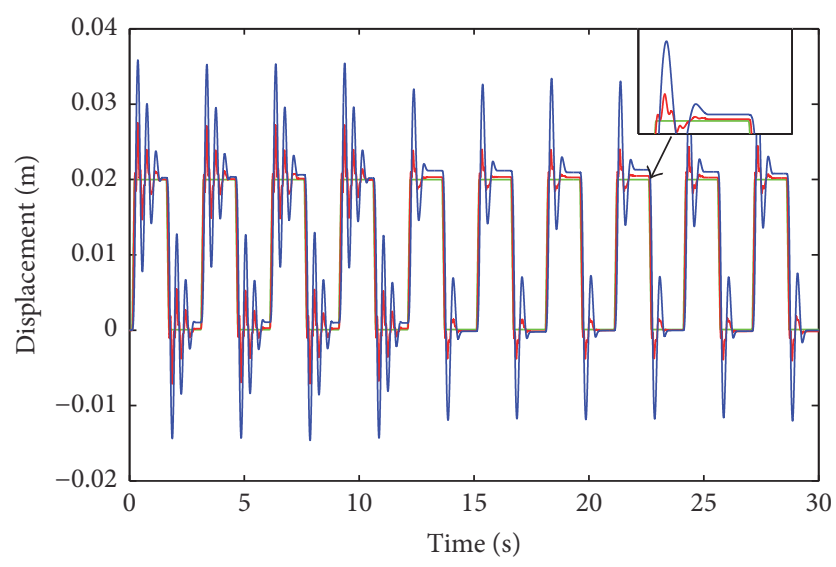

Figure 10: Transient response practice open loop (0-12s) and closed-loop (12.01-30 s).

The system was subject to the same conditions showed in the practical implementation. We can observe that the red response belonging to the chassis is affected by influence of the parametric variable in time function $\alpha(t)$. The controller does not get to eliminate this oscillations; however the designed controller has a good performance for any moment time. We can conclude that, for high frequency, the controller will have difficulty for guaranteeing good performance of system.

\section{Conclusions}

In this work a methodology was proposed to design a state derivative feedback robust gain scheduling controller that stabilizes an LTI system. In the practical implementation, the efficiency of the new technique presented was verified. The formulations of LMI with $\mathscr{D}$-stability constraints design the controller without having to invert a matrix dependent on parameter $\alpha(t)$; it is an advantage. The presented technique represented a useful tool for linear systems with derivative feedback subject to faults. The method $\mathscr{D}$-stability for allocating the eigenvalues allowed concluding the antagonistic nature of the system between the state derivative and state feedback showing how the value of the controller gains can decrease or increase, depending on how near or far from the imaginary axis the eigenvalues are.

\section{Conflicts of Interest}

The authors declare that there are no conflicts of interest regarding the publication of this paper.

\section{Acknowledgments}

The authors would like to thank the Brazilian agencies CAPES, CNPq, and FAPESP (no. 2011/17610-0) which have supported this research.

\section{References}

[1] T. H. S. Abdelaziz and M. Valášek, "Pole-placement for SISO linear systems by state-derivative feedback," IEEE Transactions on Automatic Control, vol. 151, no. 4, pp. 377-385, 2004.

[2] F. A. Faria, E. Assuno, M. C. M. Teixeira, and R. Cardim, "Robust state-derivative feedback LMI-based designs for linear descriptor systems," Mathematical Problems in Engineering, vol. 2010, Article ID 927362, 16 pages, 2010.

[3] M. C. M. Teixeira, M. R. Moreira, E. I. Mainardi Júnior et al., "Stabilizability and disturbance rejection with state-derivative feedback," Mathematical Problems in Engineering, vol. 2010, Article ID 123751, 12 pages, 2010.

[4] F. L. Lewis and V. L. Syrmos, "A geometric theory for derivative feedback," Institute of Electrical and Electronics Engineers Transactions on Automatic Control, vol. 36, no. 9, pp. 1111-1116, 1991.

[5] E. R. P. Da Silva, E. Assunção, M. C. M. Teixeira, and L. F. S. Buzachero, "Less conservative control design for linear systems with polytopic uncertainties via state-derivative feedback," Mathematical Problems in Engineering, vol. 2012, Article ID 315049, 21 pages, 2012.

[6] W. Michiels, T. s. Vyhlídal, H. Huijberts, and H. Nijmeijer, "Stabilizability and stability robustness of state derivative feedback controllers," SIAM Journal on Control and Optimization, vol. 47, no. 6, pp. 3100-3117, 2008.

[7] K. J. Astrom and B. Wittenmark, Adaptative Control, AddisonWesley, 2nd edition, 2008.

[8] J. Shamma and M. Athans, "Stabilizability and stability robustness of state derivative feedback controllers," IEEE Transactions on Automatic Control, vol. 35, no. 8, pp. 3100-3117, 1990.

[9] R. A. Hyde and K. Glover, "The application of scheduled Ho controllers to a VSTOL aircraft," IEEE Transactions on Automatic Control, vol. 38, no. 7, pp. 1021-1039, 1993.

[10] D. J. Leith and W. E. Leithead, "Survey of gain-scheduling analysis and design," International Journal of Control, vol. 73, no. 11, pp. 1001-1025, 2000.

[11] R. J. E. Merry, J. L. Holierhoek, M. J. G. Van De Molengraft, and $\mathrm{M}$. Steinbuch, "Gain scheduling control of a walking piezo actuator," IEEE/ASME Transactions on Mechatronics, vol. 19, no. 3, pp. 954-962, 2014. 
[12] V. Veselýa and A. Ilka, "Design of robust gain-scheduled PI controllers," Journal of The Franklin Institute, vol. 352, no. 4, pp. 1476-1494, 2015.

[13] V. Veselýa and A. Ilka, "Generalized robust gain-scheduled PID controller design for affine LPV systems with polytopic uncertainty," Systems \& Control Letters, vol. 105, pp. 6-13, 2017.

[14] G. Naus, Gain scheduling robust design and automated tuning of automotive controllers [Master's thesis], University of Technology Eindhoven, 2009.

[15] S. Zhang, Z. Wang, D. Ding, G. Wei, F. Alsaadi, and T. Hayat, "Improvement of power system transient stability by PV farm with fuzzy gain scheduling of PID controller," IEEE Systems Joural, vol. 11, Article ID 16841691, pp. 1684-1691, 2017.

[16] S. Zhang, Z. Wang, D. Ding, G. Wei, F. Alsaadi, and T. Hayat, "A Gain-Scheduling Approach to Non-fragile H-infinity Fuzzy Control Subject to Fading Channels," IEEE Transactions on Fuzzy Systems, 2016.

[17] P. Apkarian, P. Gahinet, and G. Becker, "Self-scheduled Ho control of linear parameter-varying systems: a design example," Automatica, vol. 31, no. 9, pp. 1251-1261, 1995.

[18] F. D. Bianchi, R. J. Mantz, and C. F. Christiansen, "Control of variable-speed wind turbines by LPV gain scheduling," Wind Energy, vol. 7, no. 1, pp. 1-8, 2004.

[19] S. Boyd, L. El Ghaoui, E . Feron, and V. Balakrishnan, Linear Matrix Inequalities in System and Control Theory, Studies in Applied Mathematics, no. 15, SIAM, Philadelphia, Pa, USA, 2nd edition, 1994.

[20] K. Tanaka, T. Ikeda, and H. O. Wang, "Fuzzy regulators and fuzzy observers: relaxed stability conditions and LMI-based designs," IEEE Transactions on Fuzzy Systems, vol. 6, no. 2, pp. 250-265, 1998.

[21] T. I. Robert, E. Skelton, and K. M. Grigoriadis, A Unified Algebric Approach to Control Design, Taylor \& Francis, Bristol, Pa, USA, 1997.

[22] L. A. Mozelli, R. M. Palhares, and G. S. C. Avellar, "A systematic approach to improve multiple Lyapunov function stability and stabilization conditions for fuzzy systems," Wind Energy, vol. 8, no. 1, pp. 1-7, 2004.

[23] Quanser, active suspension - user's manual, 2009.

[24] E. R. da Silva, E. Assunção, M. C. Teixeira, F. A. Faria, and L. F. Buzachero, "Parameter-dependent Lyapunov functions for state-derivative feedback control in polytopic linear systems," International Journal of Control, vol. 84, no. 8, pp. 1377-1386, 2011. 


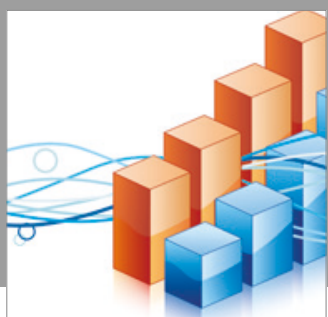

Advances in

Operations Research

vatersals

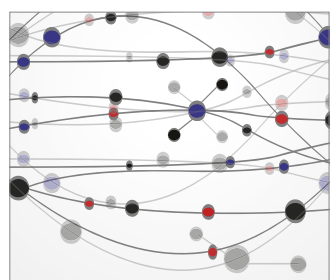

\section{The Scientific} World Journal
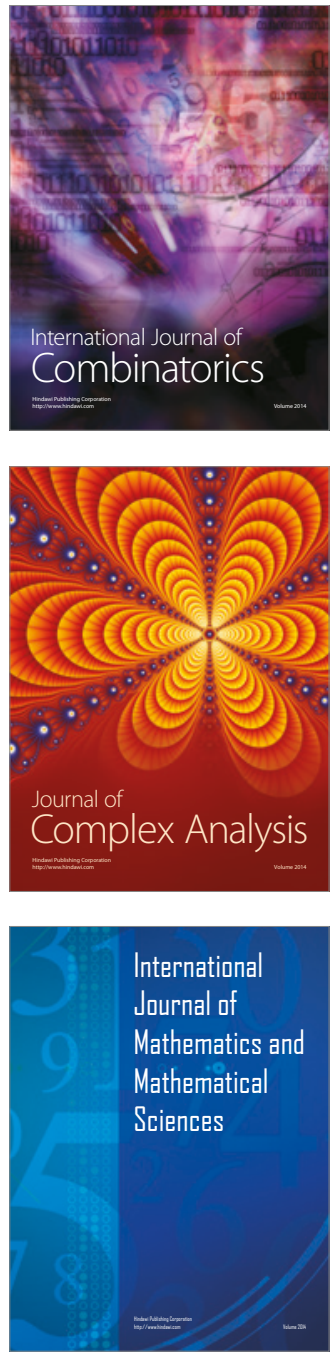
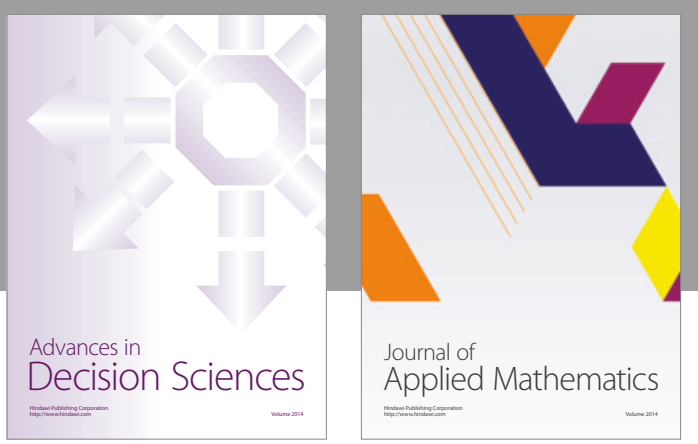

Algebra

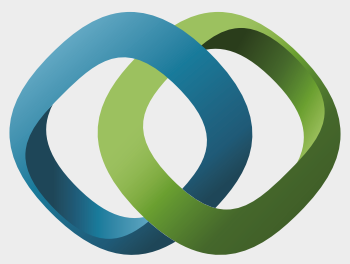

\section{Hindawi}

Submit your manuscripts at

https://www.hindawi.com
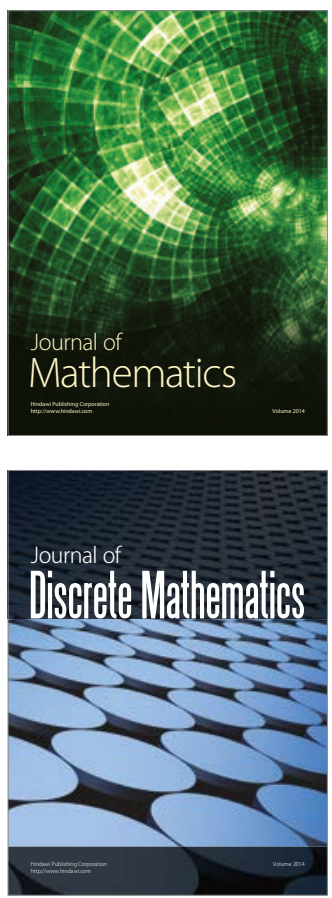

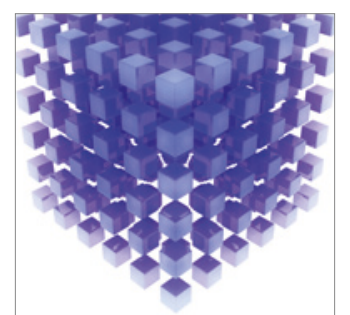

Mathematical Problems in Engineering
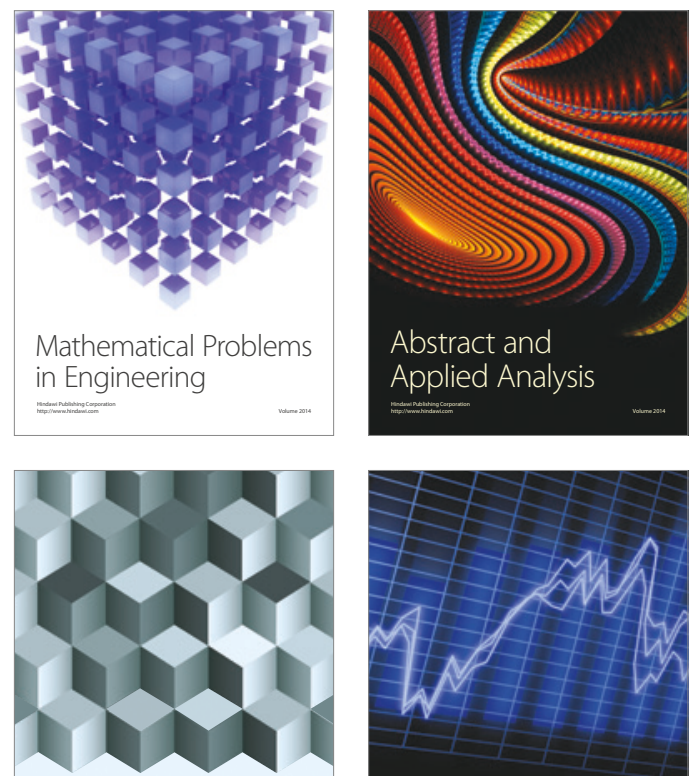

Journal of

Function Spaces

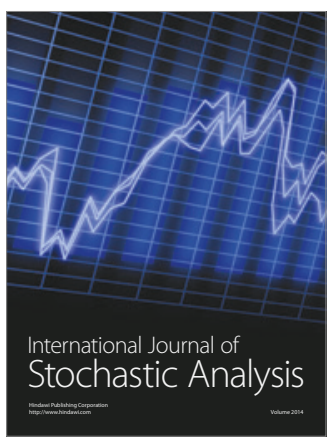

Probability and Statistics
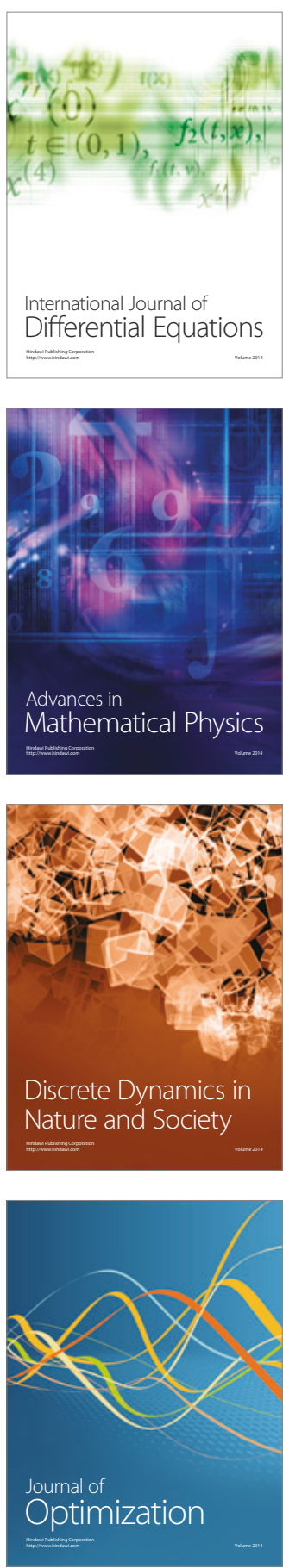